\title{
Autosomal-dominant hemochrom- atosis is associated with a mutation in the ferroportin (SLC11A3) gene
}

\author{
Giuliana Montosi, ${ }^{1}$ Adriana Donovan, ${ }^{2}$ Angela Totaro, ${ }^{3}$ Cinzia Garuti, ${ }^{1}$ Elisa Pignatti, ${ }^{1}$ \\ Stefano Cassanelli, ${ }^{1}$ Cameron C. Trenor, ${ }^{2}$ Paolo Gasparini, ${ }^{3}$ Nancy C. Andrews, ${ }^{2}$ \\ and Antonello Pietrangelo ${ }^{1}$
}

\author{
${ }^{1}$ Department of Internal Medicine, University of Modena, Modena, Italy \\ ${ }^{2}$ Division of Hematology, Howard Hughes Medical Institute, Boston, Massachusetts, USA \\ ${ }^{3}$ Telethon Institute of Genetics and Medicine, Napoli, Italy \\ Address correspondence to: Antonello Pietrangelo, Department of Internal Medicine, \\ University of Modena, Policlinico, Via del Pozzo 71, 41100 Modena, Italy. \\ Phone: 39-059-4222714; Fax: 39-059-4224363; E-mail: pietrangelo.antonello@unimo.it.
}

Received for publication June 8, 2001, and accepted in revised form July 2, 2001.

\begin{abstract}
Hemochromatosis is a progressive iron overload disorder that is prevalent among individuals of European descent. It is usually inherited in an autosomal-recessive pattern and associated with missense mutations in HFE, an atypical major histocompatibility class I gene. Recently, we described a large family with autosomal-dominant hemochromatosis not linked to HFE and distinguished by early iron accumulation in reticuloendothelial cells. Through analysis of a large pedigree, we have determined that this disease maps to $2 \mathrm{q} 32$. The gene encoding ferroportin (SLC11A3), a transmembrane iron export protein, lies within a candidate interval defined by highly significant lod scores. We show that the iron-loading phenotype in autosomal-dominant hemochromatosis is associated with a nonconservative missense mutation in the ferroportin gene. This missense mutation, converting alanine to aspartic acid at residue 77 (A77D), was not seen in samples from 100 unaffected control individuals. We propose that partial loss of ferroportin function leads to an imbalance in iron distribution and a consequent increase in tissue iron accumulation.
\end{abstract}

J. Clin. Invest. 108:619-623 (2001). DOI:10.1172/JCI200113468.

\section{Introduction}

Hereditary hemochromatosis is usually an autosomalrecessive disorder, with an estimated prevalence of up to 1 in 100 individuals in northern European populations (1). However, little was known about the molecular etiology of hemochromatosis until 1996, when it was discovered that most patients are homozygous for a unique missense mutation in HFE, a gene encoding an atypical major histocompatibility class I molecule (2). In the initial study, approximately $80 \%$ of chromosomes from hemochromatosis patients had the same mutation, converting cysteine to tyrosine at position $282(\mathrm{C} 282 Y)$ in the HFE protein. A second polymorphism, which converts histidine to aspartic acid at position 63 (H63D), is also found in a minority of patients, but this substitution is prevalent among unaffected individuals, and its role in the pathogenesis of the disease is uncertain (3).

A second gene has been found to be mutated in a small number of patients with a non- $H F E-$ linked, autosomalrecessive iron-loading disorder. These patients carry mutations in TFR2, which encodes a protein that is closely related to the transferrin receptor $(4,5)$. Despite this intriguing homology, however, the function of TFR2 has not been established, and it remains unclear how mutations in TFR2 lead to hemochromatosis.
It has become apparent that mutations in HFE and TFR2 do not account for all cases of hemochromatosis, particularly in southern Europe. A recent study of Italian patients showed that only $64 \%$ were homozygous for HFE C282Y mutations (6), and the estimated prevalence of $\mathrm{C} 282 \mathrm{Y}$ homozygosity in the general Italian population is 1 in 3,900 (7). It is likely that TFR2 mutations are much less prevalent. This suggests that mutations in other genes may cause hemochromatosis in a significant fraction of patients in southern Europe.

Recently, we described a large Italian pedigree that includes 15 patients with iron overload, resulting in hepatic fibrosis, diabetes, impotence, and arrhythmias (8). Their clinical phenotype might be confused with classical hereditary hemochromatosis, but the disorder shows autosomal-dominant inheritance, and the patients do not have mutations in HFE. Additional distinguishing features of this new clinical entity include early iron accumulation in reticuloendothelial cells and a marked increase in serum ferritin prior to elevation of the transferrin saturation. These features led us to postulate a possible defect in the recycling of red blood cell iron through the reticuloendothelial system (1). In this report we describe the results of a genome-wide screen undertaken to determine linkage for autosomal-dom- 
inant hemochromatosis. The disorder shows strong linkage to $2 \mathrm{q} 32$. One gene in the candidate region, ferroportin (also called IREG1, MTP1; refs. 9-11), is a compelling candidate for the site of the mutation causing hemochromatosis. Ferroportin is a highly conserved, multiple transmembrane-domain protein that exports iron from cells. It is expressed in several cell types that play critical roles in mammalian iron metabolism, including placental syncytiotrophoblasts, duodenal enterocytes, hepatocytes, and reticuloendothelial macrophages (9). It has been postulated to function in placental materno-fetal iron transfer, in intestinal iron absorption, and in release of iron from hepatocytes and reticuloendothelial macrophages (reviewed in ref. 12). We have found a missense mutation in ferroportin in patients with autosomal-dominant hemochromatosis that is not present in normal individuals, and we propose that this mutation is responsible for inappropriate iron loading in our patients.

\section{Methods}

Chromosomal assignment and locus identification. Human studies were approved by an institutional review board at the University Hospital of Modena (Modena, Italy). DNA was extracted from peripheral blood samples from the proband and affected relatives using a DNA blood extraction kit (Qiagen SPA, Milano, Italy) and used for a genome-wide search performed with the ABI PRISM Linkage Mapping Set (PE Biosystems, Milano, Italy). This panel is characterized by over 375 markers that define a $10-\mathrm{cM}$ resolution human index map. PCR reactions were run according to the manufacturer's instructions using fluorescently labeled primers. An aliquot of each PCR reaction was electrophoresed through an ABI PRISM 377 DNA sequencer, and results were processed using GeneScan software (PE Biosystems). Allele assignments were made using the Genotyper software package provided by the manufacturer. Statistical analysis was carried out using the assumption of an autosomal-dominant disease with complete penetrance. The disease-gene frequency was set to 0.012 , and all marker alleles were considered to be equally frequent. Two-point linkage analysis was performed using the MLINK program version 5.1 from the LINKAGE computer package (courtesy of J. Ott, New York, New York, USA). Values for maximum lod scores were calculated with the ILINK program from the same computer package.

Mutation detection. To screen for mutations, each exon of the ferroportin gene was amplified by PCR from patient and control samples and subjected to sequence analysis by the Howard Hughes Biopolymer Facility at Harvard Medical School (Boston, Massachusetts, USA). The only difference between patient and control samples was found in exon 3, after PCR amplification with a set of primers complementary to intron flanking regions (forward primer: 5'-CCTTTTGATAAGGAAGCAACTTCC-3'; reverse 5'-CAGAGGTAGCTCAGGCATTGGTCC- $\left.3^{\prime}\right)$. Patients, but not controls, were heterozygous for a single nucleotide $(\mathrm{C} \rightarrow \mathrm{A})$ substitution. To test the possibility that this change was a polymorphism, highmolecular-weight DNA was obtained from blood samples from 100 unrelated blood donors living in the region where the proband's family originated and 25 unaffected members of the proband's family. The mutation itself did not create or destroy a restriction endonuclease site. Therefore, to facilitate analysis of large numbers of samples, the portion of exon 3 containing the mutation was amplified by PCR with the Expand High Fidelity PCR system (Roche, Monza, Italy) using a mismatched sense primer (5'-GGAAGACATCATCGGT- $3^{\prime}$ ) that introduces an informative restriction site for MboII into the mutated allele. The antisense primer was the same as that used for sequencing. The PCR product was digested with MboII (MBI Fermentas GmbH, St. Leon-Rot, Germany), and resulting DNA fragments were fractionated on a $12 \%$ polyacrylamide gel and detected with ethidium bromide.

\section{Results}

The clinical phenotype of patients with autosomaldominant hemochromatosis was described in detail in an earlier report (8). The pedigree is shown in Figure 1. We determined linkage by performing a genome-wide screen, analyzing 375 markers at approximately $10-\mathrm{cM}$ intervals. We initially detected evidence of linkage for

Table 1

Lod scores between dominant hemochromatosis and chromosome 2 markers

\begin{tabular}{|c|c|c|c|c|c|c|c|c|c|}
\hline \multirow[t]{2}{*}{ Markers } & & & & $\mathrm{Z}$ at & $\Theta_{\max }$ & & & & \\
\hline & 0.00 & 0.01 & 0.05 & 0.1 & 0.2 & 0.3 & 0.4 & $Z_{\max }$ & $\left(\theta_{\max }\right)$ \\
\hline D2S2257 & 0.20 & 0.20 & 0.19 & 0.16 & 0.11 & 0.06 & 0.02 & 0.20 & $(0.000)$ \\
\hline D2S364 & $-\propto$ & 3.46 & 3.82 & 3.67 & 3.00 & 2.08 & 0.99 & 3.82 & $(0.040)$ \\
\hline D2S350 & $-\propto$ & 2.82 & 3.16 & 2.99 & 2.31 & 1.44 & 0.53 & 3.16 & $(0.040)$ \\
\hline D2S152 & 5.88 & 5.78 & 5.38 & 4.85 & 3.74 & 2.51 & 1.16 & 5.88 & $(0.000)$ \\
\hline D2S118 & 5.99 & 5.89 & 5.49 & 4.96 & 3.84 & 2.60 & 1.23 & 5.99 & $(0.000)$ \\
\hline D2S280 & $-\propto$ & 2.35 & 2.77 & 2.70 & 2.22 & 1.53 & 0.70 & 2.78 & $(0.060)$ \\
\hline D2S315 & 3.46 & 3.41 & 3.17 & 2.87 & 2.22 & 1.48 & 0.66 & 3.46 & $(0.000)$ \\
\hline D2S117 & $-\propto$ & 2.69 & 3.09 & 3.00 & 2.47 & 1.71 & 0.76 & 3.09 & $(0.050)$ \\
\hline
\end{tabular}

$-\propto$, indicates the presence of several recombinants. 


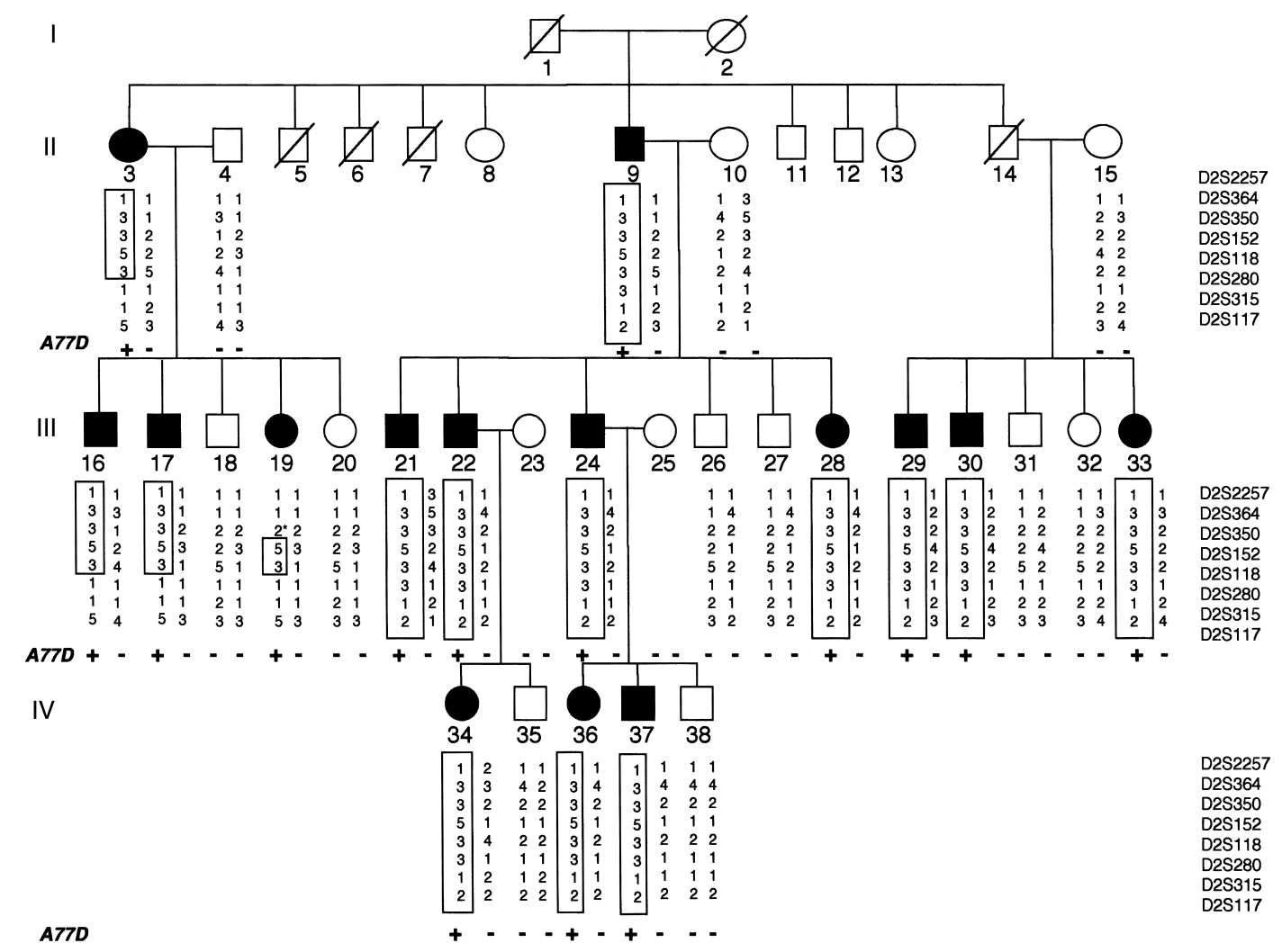

Figure 1

Pedigree of the family harboring the ferroportin A77D mutation. The large pedigree used for genetic mapping is shown. Some individuals from this family were described previously (1). Filled symbols represent affected individuals; open symbols represent unaffected individuals. All patients had increased transferrin saturation and serum ferritin and most had disease-related clinical complications. One patient, III-19, was reported previously to be unaffected (8). However, she subsequently entered menopause and developed a markedly elevated serum ferritin level; she is currently on phlebotomy treatment. Haplotypes are defined by eight microsatellite markers (on the right) spanning $2 q 32$, where SLC11A3 lies. Allelic variants have been arbitrarily numbered; boxed numbers indicate the region carrying the disease gene. Asterisks indicate recombination events. The presence or absence of the A77D mutation is indicated for each individual at the bottom of the allele columns (+, present; -, absent).

two markers (D2S364 and D2S117) from 2q32. We further analyzed this region by typing with additional markers (D2S2257, D2S350, D2S152, D2S118, $\mathrm{D} 2 \mathrm{~S} 280$, and D2S315). The disease-gene frequency was set to 0.012 , and all marker alleles were considered to be equally frequent. Pairwise linkage analysis showed maximum lod scores of 5.99 with marker D2S118 and 5.88 with marker D2S152 at a recombination frequency of 0.0 (Table 1). Significant but lower lod scores were also obtained with several of the surrounding markers. No other chromosomal regions showed evidence of linkage. The recombinants define a region of approximately 5 cM (Figure 1). Most of the known genes within this region have no apparent connection to iron homeostasis. However, one compelling candidate gene, SLC11A3 (encoding ferroportin, also known as IREG1, MTP1; GenBank accession no. NM_014585), is located within the candidate interval (9-11). The SLC11A3 gene was analyzed by amplifying each exon and determining their DNA sequences. All affected patients were heterozygous for a $\mathrm{C} \rightarrow \mathrm{A}$ substitution in exon 3 that results in replacement of alanine 77 , a small, hydrophobic amino acid, with aspartate, a large, negatively charged amino acid (A77D; Figure 2). A77 lies close to the first predicted transmembrane domain and is conserved across vertebrate species (9). This mutation modifies a predicted myristylation site and may affect the secondary structure of the protein (PHDsec software analysis, not shown). The A77D substitution was not found in 25 healthy family members or in 100 normal blood donors from the same geographic area.

We attempted to compare the function of wild-type and A77D ferroportin molecules using a Xenopus oocyte export assay described previously (9). It appeared that the A77D protein had reduced export activity, but the assay was not robust enough to provide a quantitative comparison of A77D versus wildtype activities (data not shown). We compared subcellular localization of A77D and wild-type forms of ferroportin in transfected cells and found that they localized similarly (data not shown).

\section{Discussion}

Ferroportin is the third protein to be put forward as a site of defects in patients with hemochromatosis. Mutations have also been described in HFE (2) and in 
a

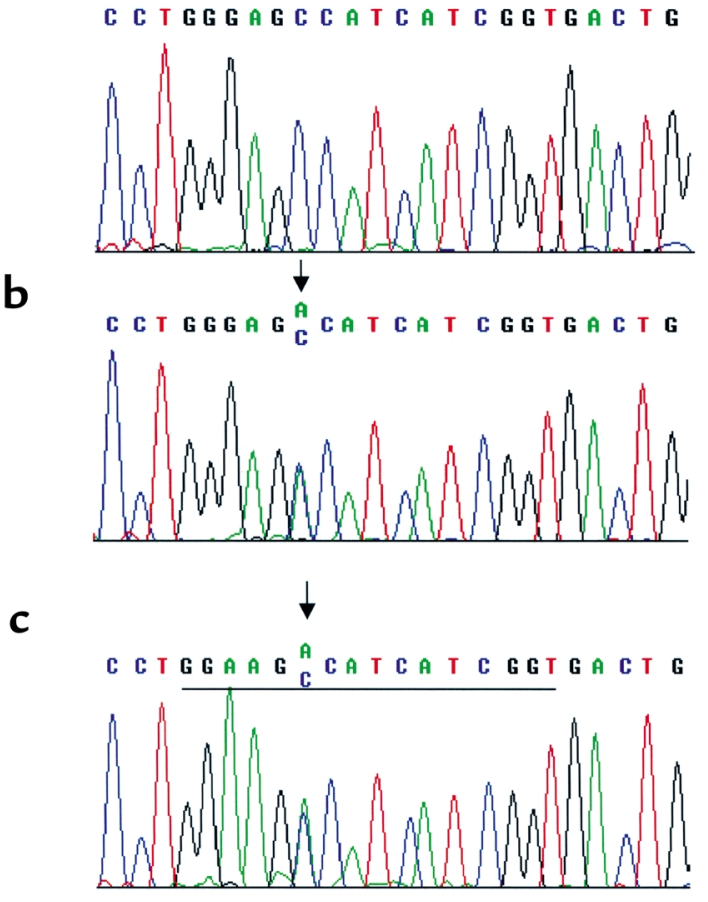

d

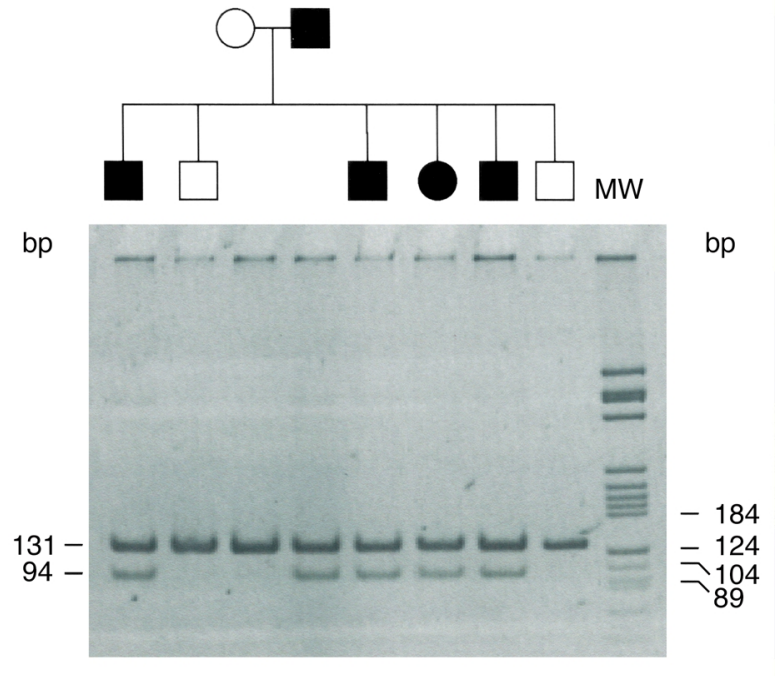

Figure 2

Mutation detection. (a-c) Sequencing chromatographs of the forward sequence of exon 3 spanning the GCC $\rightarrow$ GAC mutation (A77D) substitution are compared for a normal control $(\mathbf{a})$ and two patients $(\mathbf{b}$ and $\mathbf{c})$. The mutation is indicated by an arrow. The sequence of the mismatched primer used to introduce an informative restriction site for Mboll into the mutated allele is also indicated. The primer contains an $A \rightarrow G$ substitution, which, in the presence of the mutation, creates a novel GAAGA recognition site for Mboll. (d) Segregation of the A77D mutation, introduction of the Mboll site into mutant alleles, and restriction digestion with Mboll. The information is from individuals II-9, II10, III-21, III-22, III-24, III-26, III-27, and III-28. Normally, one fragment is obtained (131 bp). The amplification product from the mutant allele contains the novel Mboll site resulting in cleavage into 37- and 94-bp fragments. The 37-bp fragment has run off the bottom of the gel.

TFR2 $(4,5)$. Although mammalian iron metabolism is incompletely understood, the potential roles of ferroportin are more obvious than those of HFE and TFR2 (discussed in ref. 12). The normal HFE protein somehow acts in a regulatory fashion to attenuate intestinal iron absorption. HFE forms a high-affinity complex with the transferrin receptor and decreases its affinity for transferrin (13-15), but the in vivo consequences of that interaction have not been worked out. Even less is known about TFR2. It is highly expressed in the liver, and it binds to transferrin (16), but the transferrin-binding affinity is much lower than that of the classic transferrin receptor $(17,18)$, and it is not clear that TFR2 functions as a transferrin receptor in vivo. Importantly, it does not form a complex with HFE (18). It is not known whether HFE and TFR2 function in the same regulatory pathway or in distinct pathways to modulate iron absorption.

In contrast, it is easy to envision how a mutation in ferroportin might lead to iron overload. First, a gainof-function mutation might augment transfer of iron across the absorptive epithelium of the intestine, resulting in increased accumulation of dietary iron. This model is most consistent with the dominant inheritance of this disorder. However, our data are more consistent with the possibility that the mutation results in a loss of protein function. In that case, assuming that a decrease in ferroportin activity is not limiting for intestinal iron transfer, a loss-of-function mutation could lead to a perturbation of iron distribution within the body. It might cause a mild but significant impairment of iron recycling by reticuloendothelial macrophages, which normally must process and release a large quantity of iron derived from the lysis of senescent erythrocytes (discussed in ref. 12). In that case, iron retention by macrophages could lead to decreased availability of iron for the hematopoietic system and other tissues. This, in turn, might result in activation of feedback mechanisms to increase intestinal absorption, leading to iron overload. This latter model is consistent with the finding that patients with the A77D mutation in ferroportin have much larger reticuloendothelial iron stores than do patients with other forms of hemochromatosis. Although the patients with A77D mutations are not anemic, indicating that adequate iron is available for normal erythropoiesis, several were anemic earlier in life, prior to the appearance of elevated serum ferritin levels. Furthermore, several patients showed a reduced tolerance to phlebotomy and became anemic upon therapy, despite persistently elevated serum ferritin values (A. Pietrangelo, unpublished observations). These observations are consistent with 
the hypothesis that tissue iron accumulation may compensate for decreased efficiency of iron recycling, resulting in normal hematological parameters.

In summary, we propose that the A77D mutation in ferroportin impairs its activity, leading to abnormal iron homeostasis and resulting in the development of iron overload. Further insight into the pathogenesis of autosomal-dominant hemochromatosis awaits the description of additional mutations and better assays for ferroportin function in vitro and in vivo. In the meantime, sequence analysis of SLC11A3 should be added to the diagnostic armamentarium for patients with hemochromatosis not attributable to mutations in HFE or TFR2.

\section{Acknowledgments}

This work was supported by Telethon grant E-609 and European Community grant QLG1-CT-1999-00665 to A. Pietrangelo. N.C. Andrews is an Associate Investigator of the Howard Hughes Medical Institute.

1. Merryweather-Clarke, A.T., Pointon, J.J., Jouanolle, A.M., Rochette, J., and Robson, K.J. 2000. Geography of HFE C282Y and H63D mutations. Genet. Test. 4:183-198.

2. Feder, J.N., et al. 1996. A novel MHC class I-like gene is mutated in patients with hereditary haemochromatosis. Nat. Genet. 13:399-408.

3. Beutler, E. 1997. The significance of the $187 \mathrm{G}$ (H63D) mutation in hemochromatosis. Am. J. Hum. Genet. 61:762-764.

4. Camaschella, C., et al. 2000. The gene TFR2 is mutated in a new type of haemochromatosis mapping to 7q22. Nat. Genet. 25:14-15.
5. Roetto, A., et al. 2001. New mutations inactivating transferrin receptor 2 in hemochromatosis type 3. Blood. 97:2555-2560.

6. Piperno, A., et al. 1998. Heterogeneity of hemochromatosis in Italy. Gastroenterology. 114:996-1002.

7. Cassanelli, S., et al. 2001. Frequency and biochemical expression of C282Y/H63D hemochromatosis (HFE) gene mutations in the healthy adult population in Italy. J. Hepatol. 34:523-528.

8. Pietrangelo, A., et al. 1999. Hereditary hemochromatosis in adults without pathogenic mutations in the hemochromatosis gene. N. Engl.J. Med. 341:725-732

9. Donovan, A., et al. 2000. Positional cloning of zebrafish ferroportin1 identifies a conserved vertebrate iron exporter. Nature. 403:776-781.

10. McKie, A.T., et al. 2000. A novel duodenal iron-regulated transporter, IREG1, implicated in the basolateral transfer of iron to the circulation. Mol. Cell. 5:299-309.

11. Abboud, S., and Haile, D.J. 2000. A novel mammalian iron-regulated protein involved in intracellular iron metabolism. J. Biol. Chem. 275:19906-19912.

12. Andrews, N.C. 2000. Iron homeostasis: insights from genetics and animal models. Nat. Rev. Genet. 1:208-217.

13. Parkkila, S., et al. 1997. Association of the transferrin receptor in human placenta with HFE, the protein defective in hereditary hemochromatosis. Proc. Natl. Acad. Sci. USA. 94:13198-13202.

14. Feder, J.N., et al. 1998. The hemochromatosis gene product complexes with the transferrin receptor and lowers its affinity for ligand binding. Proc. Natl. Acad. Sci. USA. 95:1472-1477.

15. Lebron, J.A., et al. 1998. Crystal structure of the hemochromatosis protein HFE and characterization of its interaction with transferrin receptor. Cell. 93:111-123.

16. Kawabata, H., et al. 1999. Molecular cloning of transferrin receptor 2. A new member of the transferrin receptor-like family. J. Biol. Chem. 274:20826-20832.

17. Kawabata, H., et al. 2000. Transferrin receptor 2 -\{alpha\} supports cell growth both in iron-chelated cultured cells and in vivo. J. Biol. Chem. 275:16618-16625.

18. West, A.P., Jr., et al. 2000. Comparison of the interactions of transferrin receptor and transferrin receptor 2 with transferrin and the hereditary hemochromatosis protein HFE. J. Biol. Chem. 275:38135-38138. 